Editor Científico: Prof. Dr. Martinho Isnard Avaliação: Double Blind Review pelo SEER/OJS

Revisão: Gramatical, normativa e de formatação

\section{PLANEJAMENTO DE ADOÇÃO DE TECNOLOGIAS DE INFORMAÇÃO MÓVEIS EM EQUIPE MULTILOCALIZADA DE PROJETO}

Doi 10.21902/emprad.v1i1.250

Recebido: $15 / 08 / 2016$

Aprovado: 18/10/2016
Alex de Souza Santana ${ }^{1}$

Cristina Dai Prá Martens ${ }^{2}$

Henrique Mello Rodrigues de Freitas ${ }^{3}$

\section{Resumo}

O presente relato descreve o processo de planejamento de adoção de tecnologias de informações móveis (TIMs) em uma empresa do setor de comércio varejista de materiais para casa e construção, à luz de um modelo teórico existente na literatura. O projeto foi motivado pela demanda de implantação de uma nova loja, distante mais de 500 quilômetros da matriz. Com isso, percebeu-se que o uso de TIMs seria imprescindível para execução deste novo projeto. Como método, foi utilizado estudo de caso, por meio de abordagem qualitativa, resultando na prescrição dos recursos necessários, dos impactos esperados nos envolvidos e na organização, bem como, do ganho financeiro esperado com a adoção de TIMs. Mas, mesmo o planejamento elaborado tendo descrito os benefícios esperados com a adoção de TIMs no projeto, a proposta foi recebida com cautela pelo corpo de executivos da empresa estudada. Foram sugeridas algumas melhorias ao modelo teórico utilizado como base, que poderiam ser reafirmadas ou contrapostas em estudos futuros, principalmente no que tange o alinhamento estratégico e a intenção de adoção de determinada tecnologia por parte do indivíduo.

Palavras-chave: planejamento; tecnologias de informação móveis; projetos.

\footnotetext{
${ }^{1}$ Mestrando pela Universidade Nove de Julho - UNINOVE, São Paulo (Brasil)

E-mail: alex.santana-br@live.com

${ }^{2}$ Doutora pela Universidade Federal do Rio Grande do Sul - UFRGS, Rio Grande do Sul (Brasil)

Professora pela Universidade Nove de Julho - UNINOVE, São Paulo (Brasil)

E-mail: cristinadpmartens@gmail.com

${ }^{3}$ Doutorado pela Universite Pierre Mendes France Ecole Superieure des Affaires - UPMF, (França)

Professor pela Universidade Nove de Julho - UNINOVE, São Paulo (Brasil)

E-mail: freitas138@gmail.com
} 


\begin{abstract}
This report describes the planning process of adoption of mobile information technologies (MITs) at a company of the retail trade sector of materials for building and construction, in the light of an existing theoretical model in the literature. The project was motivated by the demand for implementation of a new store, far more than 500 kilometers from the matrix. Thus, it was noticed that the use of MITs would be essential to implementing this new project. As a method, case study was used, through a qualitative approach, resulting in the prescription of the required resources, expected impacts on stakeholders and organization, as well as the financial gain expected with the adoption of MITs. The elaborated planning described the expected benefits of the adoption of MITs on the project, but even so, the proposal was met with caution by the executive body of the company studied. It is suggested some improvements to the theoretical model used as a basis, which could be reaffirmed or opposed in future studies, especially regarding the strategic alignment and the intention of adopting certain technology by the individual.
\end{abstract}

Keywords: planning; mobile information technology; projects. 


\section{Introdução}

Uma empresa jovem do setor de varejo de materiais para casa e construção, integrante um grupo multinacional francês com mais de 450 anos de história, adotou em 2015 a gestão de projetos como estratégia para atender suas necessidades de expansão comercial. Esta empresa, que aqui será denomida Alpha, até então tinha sua atuação focada no estado de São Paulo, com média de abertura de apenas uma nova loja a cada dois anos. Mas, a demanda de novas lojas cresceu movida pela competição e necessidade de posicionamento no mercado. Somente entre o segundo semestre de 2015 e primeiro semestre de 2016, previa-se a abertura de sete novas lojas. Outrora, conforme dito por Carvalho e Rabechini (2003), as empresas estão vivendo um processo de transformação, organizando-se para dar respostas eficazes e rápidas, refletindo sua competência em aproveitar oportunidades, respeitando as limitações de tempo, custo e especificações. Nota-se que com a empresa Alpha, não estava sendo diferente.

Seguia-se normal a implantação das ferramentas e técnicas de gestão de projetos, até que uma novidade trouxe muitas interrogações para o corpo de diretores, em especial à diretoria de operações, responsável pela implantação de novas lojas, e também aos gestores funcionais envolvidos em projetos. A novidade era que, uma nova unidade precisaria ser aberta, mas não em São Paulo, e sim, Belo Horizonte - Minas Gerais. Algumas das perguntas que surgiram, foram e não se limitaram a: como gerir este projeto sem fazer com que os membros da equipe fiquem longos períodos fora da unidade principal e de suas atribuições funcionais? Ficará muito mais caro deslocar os membros das equipes para locais muito distantes, haja vista o surgimento de custos adicionais com passagens aéreas, alimentação, translados e estadias, seria possível mitigar esse custo adicional? Como garantir que se mantenha clara a comunicação e integração entre os membros da equipe?

$\mathrm{O}$ uso de tecnologias informação móveis (TIMs), onde se incluem os dispositivos portáteis e sistemas de informação, que permitem comunicação e acesso a dados, como laptops e smartphones, tem crescido cada vez mais (Saccol \& Reinhard, 2007). A aplicação dessas tecnologias nos diversos processos organizacionais denomina-se mobilidade empresarial (Sorensen, 2011). Observou-se de imediato a possibilidade de utilização de TIMs como uma alternativa estratégica para atendimento da necessidade surgida. O que não se sabia de imediato, era como então, planejar a adoção do uso de TIMs para este novo projeto e como medir o sucesso da adoção da estratégia.

Este relato descreve o processo de elaboração de um planejamento de adoção de TIMs no projeto de implantação da nova loja da empresa Alpha em Minas Gerais, tendo como base o modelo teórico de planejamento de iniciativa de adoção de tecnologias móveis, proposto por Freitas e Machado (2009). Espera-se contribuir com a teoria existente ao explicitar-se a aplicabilidade em gestão de projetos do modelo teórico utilizado, visto ser um modelo destinado à adoção de TIMs no âmbito organizacional em geral, bem como, possíveis lacunas existentes, que possam instigar melhorias no modelo e/ou pesquisas futuras. No que tange a contribuição prática, espera-se que os resultados obtidos com o uso de TIMs nesse projeto possam estimular a adoção de tal estratégia nos projetos futuros, não só da empresa Alpha, como quaisquer empresas que possuam características de mobilidade em seus projetos.

Seguindo o protocolo para elaboração de relatos de produção técnica (Biancolino, Kniess, Maccari, \& Rabechini Jr., 2012) os tópicos seguintes incluirão: o referencial teórico dos construtos envolvidos, a metodologia adotada neste relato, a apresentação dos resultados obtidos com respectiva análise, e por fim, as conclusões e considerações finais dos autores. 


\section{Referencial: adoção de TIMs em equipes de projetos}

Projetos consistem de um esforço temporal, objetivando a criação de um produto único, serviço ou resultado exclusivo. A gestão de projetos, por sua vez, refere-se à aplicação de habilidades, técnicas, ferramentas e conhecimentos, para que se atinjam os requisitos definidos para o projeto. Essa diferença pode ser distinguida ao se observar as definições estabelecidas pelo Project Management Institute - PMI na $5^{\text {a }}$ edição do guia Project Management Body of Knowledge - PMBoK (PMI Standards Committee, 2013) e pela norma International Organization for Standardization - ISO 21500 (ISO, 2012).

Projetos, independentes de sua natureza e complexidade, são executados por equipes. Em seu artigo e livro, Katzenbach e Smith (2005) definem que equipe é um grupo de pessoas com aptidões complementares, comprometidas com um objetivo comum, que realizam trabalho interdependente e são coletivamente responsáveis pelos resultados. Thamhain (2012) define equipe de projeto como sendo um conjunto de indivíduos, selecionados por suas habilidades específicas e suas qualidades.

Muitas vezes, os membros do grupo têm diferentes necessidades, origens e experiências que devem ser habilmente focadas e gerenciadas para transformar o grupo de trabalho em uma equipe integrada e unificada. Ebrahim, Ahmed e Taha (2009) definiram equipe virtual como um grupo temporário de trabalhadores do conhecimento dispersos geograficamente, organizacionalmente e/ou no tempo, os quais coordenam seu trabalho predominantemente com tecnologias da informação e comunicação a fim de realizar uma ou mais tarefas organizacionais. Equipes móveis ou virtuais demandam o uso de recursos tecnologias de informação móveis (TIMs), para comunicação e condução das atividades.

Mobilidade está relacionada à possibilidade de levar um dispositivo de tecnologia da informação a qualquer lugar (Kakalota \& Robinson, 2002). Com base nesta definição podem ser considerados como exemplos de TIMs, os laptops, smartphones e dispositivos de conexão à internet 4G. Segundo Saccol e Reinhard (2007), o uso de TIMs permite que as atividades dos colaboradores possam ser executadas em qualquer lugar ou horário. Mas, adotar o uso de recursos de TI em organizações exige planejamento e as particularidades de cada organização devem ser levadas em conta (Albertin, 2001). Neste sentido, modelos teóricos que auxiliem o planejamento de adoção de TIMs, são de grande valia à prática.

O principal referencial teórico deste relato técnico é o modelo teórico de planejamento de iniciativas de adoção de tecnologias móveis proposto por Freitas e Machado (2009). O referido modelo abrange o contexto externo e interno à organização, os impactos nos indivíduos, na própria organização e na interação entre estes. Apesar de ser um modelo teórico voltado para o planejamento de adoção de TIMs no âmbito organizacional em geral, neste relato, o âmbito da iniciativa de adoção de tal estratégia é em um projeto específico.

Relativo ao contexto externo à organização, o modelo teórico sugere a observação de fatores regulatórios, éticos e socioculturais. Quanto ao contexto organizacional, Freitas e Machado (2009) propõem a observação dos seguintes fatores internos que podem influenciar a iniciativa de adoção de TIMs: a natureza da relação entre o indivíduo e a organização, as formas de interação disponíveis entre eles, os recursos de tecnologia da informação já disponíveis, os processos existentes, a cultura e capacidade de aprendizagem da organização.

Freitas e Machado (2009) extraem de literaturas anteriores, diversos possíveis impactos previstos no indivíduo, na organização e na relação entre indivíduo e organização. Como exemplo, e não se limitando a tais, estão: disponibilidade do indivíduo, custos financeiros, processos da organização, agilidade, produtividade e acessibilidade geográfica. 
Por fim, o modelo teórico em questão sugere as definições organizacionais, que tratam justamente das opções que a empresa pode fazer, tendo em vista as análises dos contextos externo e interno, dos indivíduos, dos impactos esperados e das interações. É possível analogamente dizer que é nas definições organizacionais que o planejamento da adoção de TIMs em si, ganha vida. Dentre as definições apontadas estão: características da interação, tecnologia, aprendizado, política, processos, custos e riscos. A Figura 01 representa o modelo teórico detalhado proposto por Freitas e Machado (2009).

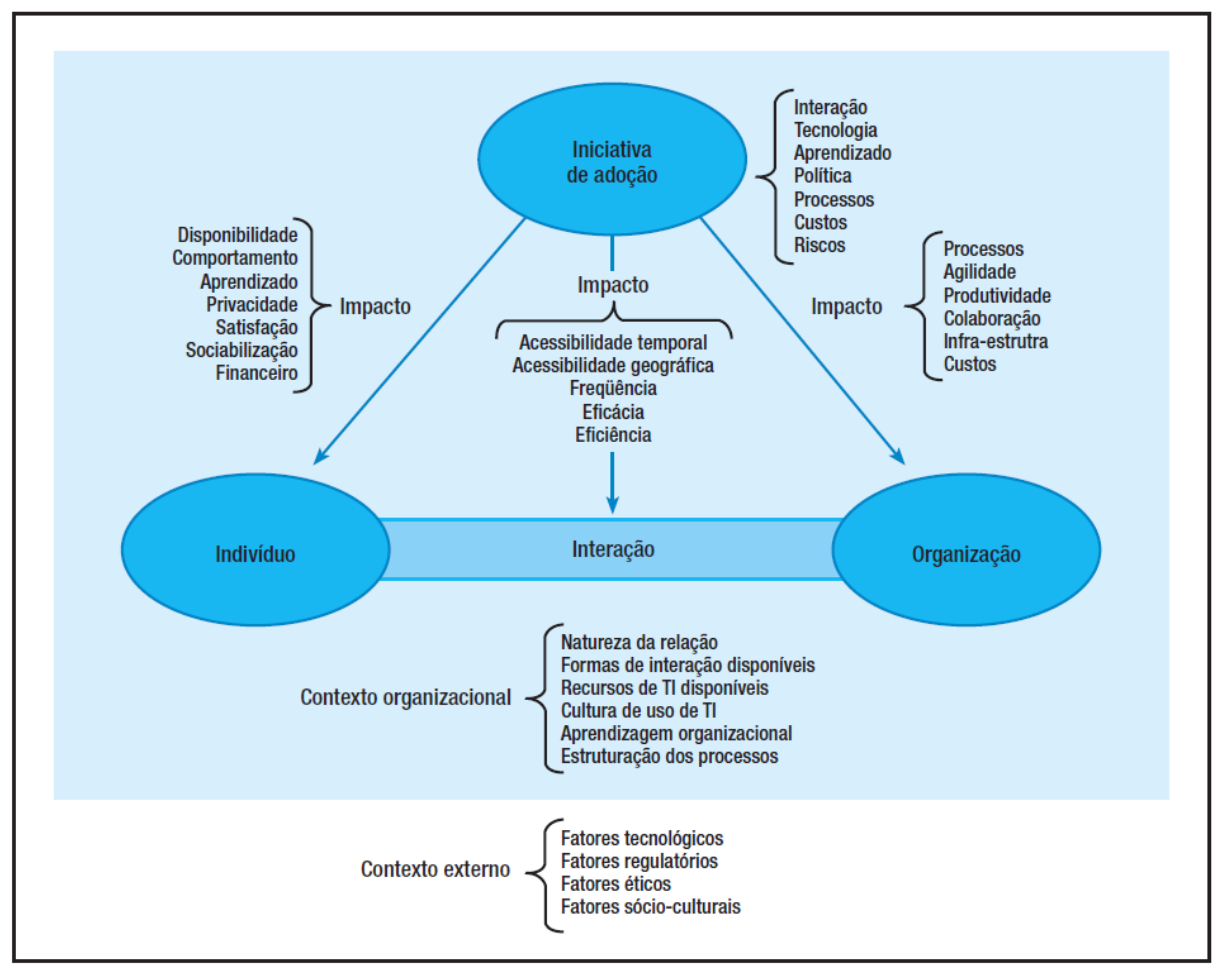

Figura 01. Modelo teórico detalhado para o planejamento de adoção de TIMs Fonte: Freitas e Machado (2009, p. 109)

\section{Metodologia}

Adotou-se uma abordagem qualitativa por meio de estudo de caso (Yin, 2014), com caráter descritivo (Sandelowski, 2000) em menor parte, porém, prescritivo por natureza de um relato técnico (Biancolino, Kniess, Maccari, \& Rabechini Jr., 2012; Van Aken, 2007). Fez-se uso de análise documental e observação direta (Patton, 2005). Documentalmente foram observados: o organograma da empresa, atas de reunião, relatórios de pedidos de reembolso por despesas de deslocamento, normas e regulamentos de conduta para uso dos recursos de informática definidos pela empresa Alpha, planilhas de estimativas de custo relativas a recursos de informática (hardware e software), contratos de serviço de dados e voz e informações disponíveis na intranet corporativa. Também foram diretamente observadas algumas reuniões de outros projetos em andamento na empresa Alpha, as atividades executadas pelos colaboradores em seus setores específicos, comportamentos e ações individuais referentes à execução das atividades a eles atribuídas em projetos existentes.

O modelo de planejamento de adoção de tecnologias móveis (Freitas \& Machado, 2009) foi o alicerce de todo o planejamento, fornecendo o direcionamento necessário para o planejador, neste caso um dos autores deste relato, onde, a cada item do referido modelo teórico, procurava observar a realidade e os registros documentais da empresa Alpha, 
permitindo a descrição dos resultados e a prescrição do planejamento para adoção das TIMs para o novo projeto a ser executado em Belo Horizonte.

Para análise do contexto externo, observou-se a infraestrutura de comunicação existente na empresa e a infraestrutura de telecomunicações disponibilizados pelas concessionárias prestadoras de serviços. Adicionalmente foram consultados contratos já firmados de prestação de serviços entre a empresa Alpha e as concessionárias, para observação de limitações de uso e/ou restrições contratuais.

O setor de Recursos Humanos da empresa Alpha foi fundamental em auxiliar o entendimento de possíveis diferenças culturais, dos regulamentos internos e das relações trabalhistas. Outro departamento com importante participação foi o de Tecnologia de Informação, fundamental para o esclarecimento dos recursos de informática e comunicação disponíveis e/ou aplicáveis, incluindo hardwares e softwares, além das estimativas de custos.

Os impactos esperados nos indivíduos, na organização e entre eles, foram originados basicamente de literatura existente (Freitas e Machado, 2009), estando estes possíveis fatores de impacto citados no próprio modelo teórico utilizado como base. A observação direta neste quesito foi de grande importância para entender as ações e comportamentos atuais e vislumbrar então, os possíveis impactos sugeridos na literatura base.

\section{Resultados obtidos}

Nos subtópicos seguintes serão exploradas cada uma das dimensões sugeridas no modelo teórico proposto por Freitas e Machado (2009) e nelas explicitando-se a realidade observada no contexto da empresa Alpha, assim como as prescrições resultantes.

\subsection{Contexto Externo}

Os membros do projeto estariam se locomovendo na capital de São Paulo e na capital de Minas de Gerais. No que se referem aos fatores tecnológicos, ambas as localidades dispõem de rede de dados celular com tecnologia $4 \mathrm{G}$ e redes cabeadas. A comunicação por voz também é viável via rede celular, em todas as frequências de operação homologadas pela Anatel. Portanto, a infraestrutura de comunicação e conexão em ambas as localidades atendem a qualquer opção de dispositivos móveis que venha a ser adotada, não oferecendo maiores riscos à adoção de estratégia de comunicação móvel.

Com relação aos fatores regulatórios, devido à impossibilidade de mensurar o nível de uso do tráfego de dados e voz, faz-se necessário assegurar que o contrato a ser realizado com as operadoras destes serviços, não imponham limitações de uso. Além disso, no intuito de evitar situações trabalhistas adversas, todo o custo de operação do uso destas tecnologias deverá ser absorvido pela empresa Alpha.

O bom uso dos dispositivos e ferramentas, bem como do comportamento desejado pelos usuários, deverá ser assegurado por meio de política de uso da tecnologia da informação, já existente no grupo detentor da empresa Alpha, devendo o mesmo ser preferencialmente traduzido para o idioma português brasileiro.

No que se referem aos fatores éticos, estes também poderão ser assegurados pela política de uso da tecnologia da informação, sendo ainda reforçados pelas políticas comuns a que estão sujeitos todos os colaboradores da empresa Alpha. 
Por fim, os fatores socioculturais são identificados nos membros da equipe do projeto, que são de diferentes gerações e com diferentes níveis de habilidades computacionais. Esperase então, imposições de barreiras por parte de algumas pessoas e forte motivação por parte de outras. Este equilíbrio ocorrerá naturalmente e com a percepção dos membros do projeto a cerca dos benefícios do uso das tecnologias móveis e sistemas.

\subsection{Contexto Organizacional}

A natureza da relação é comum entre empregador e empregados. Os membros da equipe do projeto, incluindo o gerente do projeto, são contratados de acordo com as regras estabelecidas na Consolidação das Leis do Trabalho - CLT. As principais formas de interação existentes atualmente são por telefone, e-mail e reuniões face-a-face.

Com relação aos recursos de TI disponíveis, laptops são usados por alguns membros da equipe do projeto, porém, destes, nem todos possuem acesso à internet via modem $4 \mathrm{G}$ ou similar. Na matriz da empresa Alpha, existe disponibilidade de acesso à internet via cabo para todos, independentemente do tipo de equipamento utilizado. Os aparelhos de comunicação celular estão disponíveis para todos, sem limite de utilização, com apenas uma exclusiva exceção, de um membro da equipe do setor Engenharia.

Não há significante diferença cultural no que tange a nacionalidade ou naturalidade dos membros da equipe de projeto. Há de se levar em consideração apenas a forte departamentalização da empresa. Os membros são muito focados nas atividades referentes aos seus setores específicos (atividades funcionais), não tento ainda, uma visão conjunta de objetivo, de equipe de projeto propriamente dita.

Por fim, sobre a aprendizagem, observou-se que membros da equipe de projeto demonstram facilidade na aprendizagem de novos processos e adaptação a estes. Não é esperado grande impacto na estrutura dos processos de gestão de projetos que estão sendo implantados na empresa Alpha, mas apenas na forma de interação dos membros da equipe, por meio das tecnologias móveis e ferramentas que serão adotadas.

\subsection{Impactos previstos nos indivíduos}

Os indivíduos citados são os membros da equipe do projeto. Espera-se que não haja impacto em suas vidas particulares, ou seja, que os mesmo façam uso das tecnologias móveis e ferramentas para fins de realização de suas atividades pertinentes ao projeto, apenas nos horários a isto designados. Acrescenta-se possibilidade de maior sociabilização e senso de equipe, visto a disponibilidade integral de informações do projeto como um todo, bem como da satisfação com o uso de novos recursos. Outros impactos importantes esperados são as reduções no número de longas viagens e da ausência prolongada das atividades funcionais causadas pelos deslocamentos. Todo o custo relativo à adoção de tecnologias móveis para o projeto deve ser absorvido pela empresa Alpha.

\subsection{Impactos previstos na organização}

Dentre aos impactos previstos na empresa Alpha estão o ganho na agilidade de tomada de decisões e acesso às informações e melhor colaboração entre os membros. Mas, o fator de 
maior destaque é a possibilidade de ganho financeiro resultantes da redução da necessidade de mobilização de colaboradores para outro Estado, além da redução da necessidade de que os mesmos colaboradores fiquem longos períodos afastados de suas atividades funcionais.

\subsection{Impactos previstos na interação entre indivíduo e organização}

Acessibilidade às informações do projeto em qualquer lugar, clareza e disponibilidade de informações para tomada de decisão, comunicação mais objetiva.

\subsection{Iniciativa de Adoção}

Considerando-se as variáveis contextuais e os impactos previstos, apresentam-se as opções que a organização poderia adotar para atendimento às necessidades do projeto da empresa Alpha, no que concerne à adoção de tecnologias móveis.

Dado o contexto de uma equipe de projeto, as interações poderiam ser iniciadas por quaisquer membros da equipe, independente de setor, posição ou forma de interação, desde que observadas às condições estipuladas no Plano de Gerenciamento da Comunicação, a ser elaborado.

Quanto à tecnologia necessária, a Tabela 01 ilustra quais e quantos dispositivos móveis deveriam ser adquiridos, levando em consideração os membros com características móveis ou fixas para o projeto. O objetivo é que todos passem a portar computadores (laptops ou desktops) com acesso a internet (via cabo ou $4 \mathrm{G}$ ), telefone celular habilitado para efetuar e receber ligações.

Tabela 01:

Aquisição de dispositivos móveis necessários

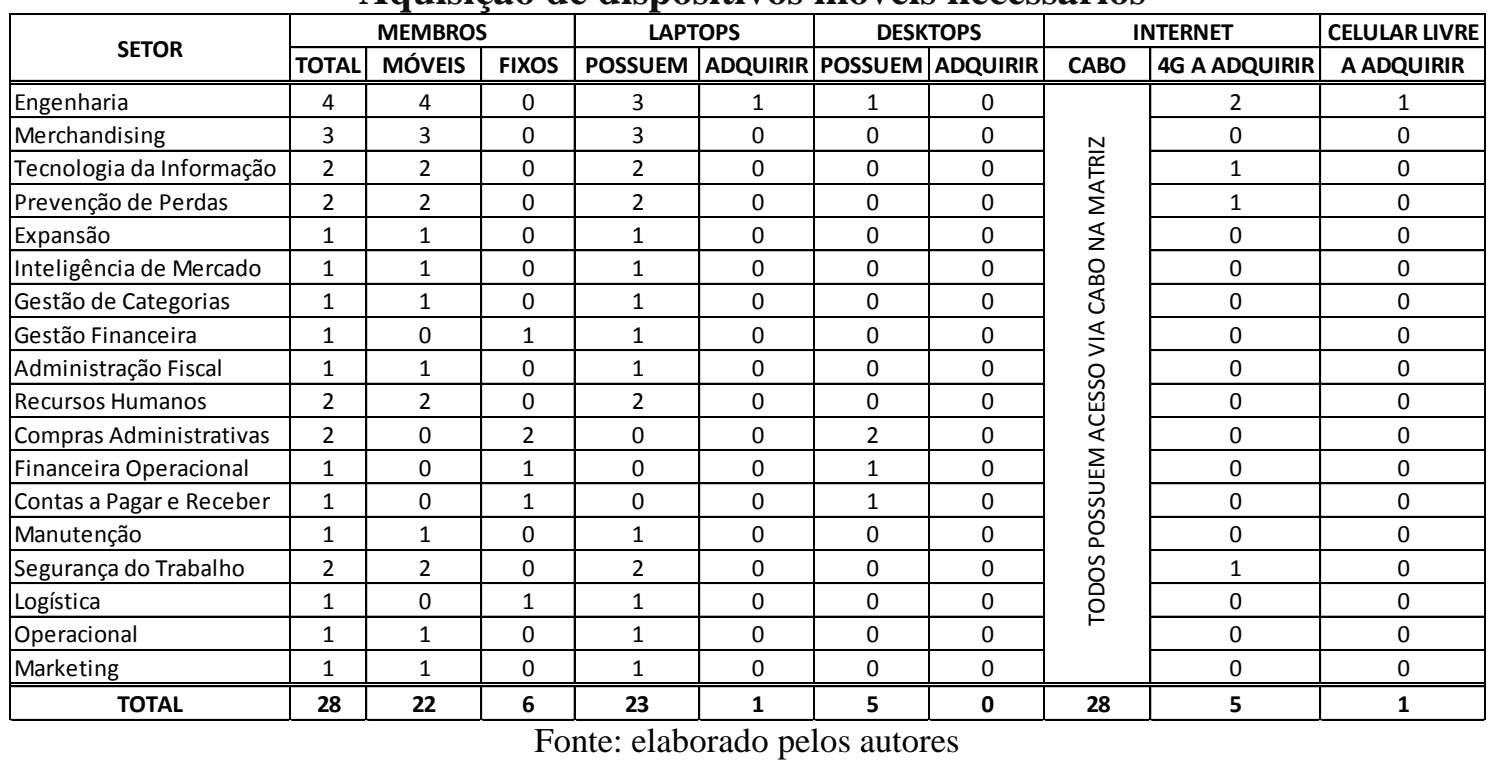

De acordo com o levantamento, faz-se necessário a aquisição de 01 (um) laptop para um dos membros móveis que ainda não o possui, integrante do setor de Engenharia, 05 (cinco) tokens de acesso à internet $4 \mathrm{G}$ e 01 (um) telefone celular livre para efetuar e receber ligações.

Quanto aos softwares, entre os que poderiam ser utilizados estão: a) Webex - Que permite criar reuniões virtuais, com voz, vídeo, compartilhamento de telas e documentos; b) 
Rede social corporativa - Trata-se de uma rede social privada que permite a criação de um grupo (comunidade virtual) específico e fechado do projeto, onde os membros poderão trocar fotos do projeto, postar comentários, consultar e reportar tarefas designadas, compartilhar resultados, etc.

Ambas as aplicações (Webex e rede social corporativa) já estão disponíveis e com acesso gratuito por meio da intranet do grupo detentor da empresa Alpha. Os demais aplicativos comuns de trabalho como, planilhas eletrônicas, leitores de PDF, editores de texto, já estão disponibilizados em todos os computadores. Quanto aos dispositivos móveis necessários, fica a cargo da empresa a disponibilização imediata destes recursos, após aprovação da adoção da estratégia de adoção das TIMs.

Sobre o aprendizado, membros do projeto serão comunicados quanto ao uso das ferramentas Webex e Rede Social Corporativa, na reunião de abertura do projeto, recebendo em seguida os manuais de utilização no formato PDF. Durante a própria reunião de abertura deve ser acordado um prazo para realização de um treinamento formal com todos os membros da equipe do projeto, a ser ministrado por membro qualificado da equipe de informática.

No que se refere à política, imediatamente após a reunião da abertura do projeto, os membros da equipe deverão receber por meio do membro designado da equipe de informática, a política de uso das ferramentas de informática já existentes no grupo, a qual todos os colaboradores já estão sujeitos. Preferencialmente, o material existente, que se encontra na língua inglesa, deverá ser traduzido, para facilitar o entendimento.

A adoção de TIMs não implicará mudanças nos processos de gestão de projetos já estabelecidos, nem ao processo existente de abertura de novas lojas. A mudança se dará efetivamente na forma de realização de reuniões e de disseminação de informações pertinentes ao projeto onde elas serão aplicadas, logo, importantes considerações deverão ser feitas no Plano de Gerenciamento das Comunicações. Esse plano deverá possuir tópicos que definam claramente: os dias e horários semanais de realização das reuniões virtuais por meio da ferramenta Webex; a colocação do gestor de projetos como cópia de qualquer e-mail entre às áreas quando o assunto for relativo ao projeto; a comunicação ao gestor do projeto de qualquer informação que tenha sido tratada entre os membros da equipe via celular; a divulgação semanal via e-mail por parte do gestor do projeto à todos os membros da equipe sobre os principais acontecimentos da semana; a postagem diária sobre o andamento do projeto na rede social corporativa (bem como fotos e documentos pertinentes).

A Tabela 02 demonstra a expectativa de custos para adoção de TIMs no projeto da empresa Alpha, com base nos requisitos tecnológicos observados, no intuito de garantir que todos os membros possuam os recursos mínimos recomendados para adoção da estratégia.

Tabela 02:

Custos esperados com a adoção de TIMs

\begin{tabular}{|c|c|c|c|}
\hline Item a aquirir & Quantidade (unid.) & Custo Unitário (R\$) & Custo Total (R\$) \\
\hline Laptop & 1 & $1.500,00$ & $1.500,00$ \\
\hline Token 4G & 5 & 80,00 & 400,00 \\
\hline Celular livre & 1 & 100,00 & 100,00 \\
\hline \multirow{2}{*}{ TOTAL } & - & $\mathbf{1 . 6 8 0 , 0 0}$ & $\mathbf{2 . 0 0 0 , 0 0}$ \\
\hline \multicolumn{3}{|c|}{ CUSTO ADICIONAL MENSAL DE MANUTENÇÃO (R\$) } \\
\hline & Internet 4G & 500,00 & \\
\cline { 2 - 3 } & Celular & 100,00 & \\
\cline { 2 - 3 } & TOTAL & $\mathbf{6 0 0 , 0 0}$ \\
\cline { 2 - 3 } & \multicolumn{2}{|c}{}
\end{tabular}

Fonte: elaborado pelos autores 
Os custos dos recursos já existentes não são considerados na Tabela, pois continuariam existindo independentes da aprovação ou não da proposta de adoção de TIMs no projeto. Portanto, aqui são apresentados somente os custos adicionais para implantação da proposta.

As ferramentas Webex, rede social corporativa, e-mails, planilhas, editores de texto, etc., já são fornecidas pelo grupo a que pertence a empresa Alpha, portanto, também não representam nenhum custo adicional para a estratégia de adoção de TIMs no projeto, bem como, a infraestrutura de acesso à internet via cabo na matriz da empresa Alpha e os recursos nela já existentes, como demais laptops, desktops e celulares.

Por fim, no que se refere aos riscos, entre os riscos iniciais identificados para a adoção de TIMs, o maior é justamente a possibilidade de restrição de tráfego de dados pela operadora de conexão móvel celular, bem como do uso dos serviços de telefonia. Portanto, a equipe de tecnologia da informação deve monitorar qualquer possibilidade de mudança no contrato de prestação de serviços que limitem futuramente a utilização das redes de dados e voz.

\section{Conclusões}

O modelo teórico para adoção de tecnologias móveis, proposto por Freitas e Machado (2009), mesmo sendo um modelo voltado para adoção de tecnologias móveis no âmbito organizacional em geral, pôde ser facilmente utilizado no contexto da gestão de projetos. Fezse necessário apenas a adaptação da expressão "indivíduos", que no modelo teórico de Freitas e Machado (2009) é entendido como qualquer pessoa interna ou externa à organização (clientes, fornecedores, empregados, etc.). Este mesmo termo, no âmbito da gestão de projetos, precisou ser entendido como qualquer membro da equipe do projeto.

Com o desenvolvimento do trabalho, percebeu-se a ausência no modelo teórico utilizado (Freitas \& Machado, 2009), de algo relacionado à análise de viabilidade. Da mesma forma, sugere-se também a inclusão de algum aspecto relacionado ao alinhamento estratégico da organização onde as TIMs serão aplicadas. De fato, na prática, se fez necessário uma apresentação do retorno esperado com a adoção da estratégia, no sentido de auxiliar a tomada de decisão go no go por parte da alta direção.

Ainda sobre o modelo teórico proposto por Freitas e Machado (2009), nele pode-se observar a consideração de dois grandes contextos, o contexto externo e o contexto organizacional. Porém, em literatura observa-se que Moore e Benbasat (1991) apresentavam em seus estudos a importância da percepção dos usuários no processo de adoção de uma nova tecnologia. Complementarmente, Bautzer (2009) afirmava que ao se compreender a percepção de novas tecnologias na ótica dos usuários diretos em potencial, pode-se identificar a aceitação e o nível de conforto proporcionado pelo recurso implantado, o que contribui para melhor aproveitamento dos benefícios esperados.

Portanto, baseado nas objeções supracitadas, sugere-se a inclusão de um terceiro contexto no modelo teórico de Freitas e Machado, o qual pode ser naturalmente denominado como contexto "individual" ligado à já existente dimensão do indivíduo, objetivando compreender a intenção de adoção das TIMs por parte do indivíduo.

A percepção do indivíduo já figura presença em diversos reconhecidos modelos teóricos sobre intenção de adoção de novas tecnologias ou de inovações, como por exemplo, o Modelo de Aceitação de Tecnologia 2 - TAM2 (Venkatesh \& Davis, 2000), a Teoria de Difusão da Inovação - IDT (Rogers, 2003) e a Teoria Unificada de Aceitação e Uso de Tecnologia - UTAUT (Venkatesh et al, 2003). 
De fato, observou-se certa motivação pela empresa Alpha em adotar o uso de TIMs, mas, ainda com certa cautela na aprovação da execução, que não ocorreu de imediato e foi tratada como um projeto piloto, e sem o investimento dos recursos mínimos sugeridos. Possível é que um dos motivos desta cautela tenha sido a estreita observação apenas dos possíveis impactos nos indivíduos, deixando de lado as variáveis que influenciam a intenção de adoção das TIMs pelos atores envolvidos, como por possíveis exemplos a facilidade de uso percebida (sugerida pelo TAM2), a expectativa de desempenho das tecnologias (conforme sugerido pela UTAUT) ou a compatibilidade (sugerida pela IDT).

A Figura 02 ilustra as possíveis melhorias sugeridas ao modelo original de Freitas e Machado (2009).

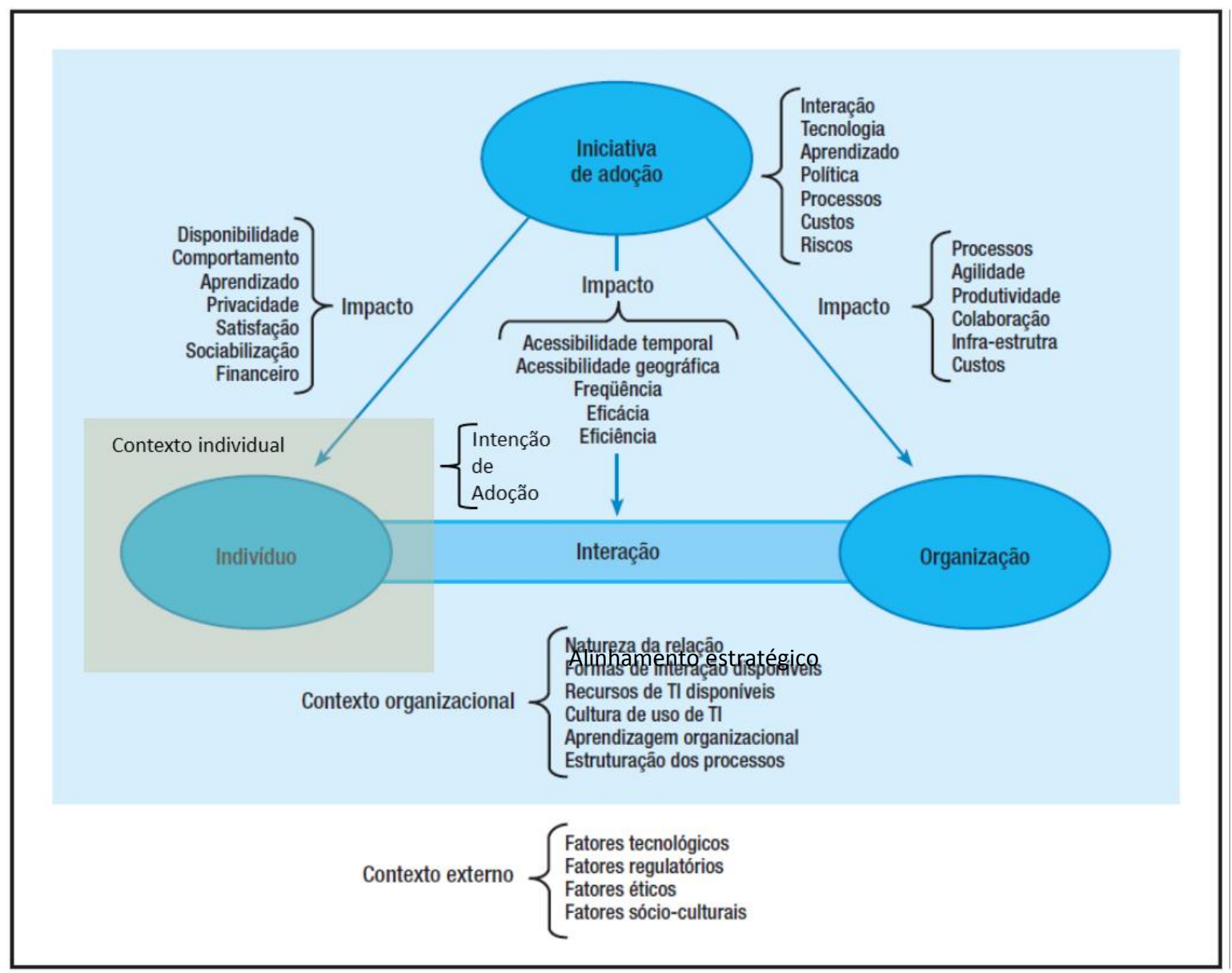

Figura 02. Melhorias sugeridas no modelo teórico detalhado para o planejamento de adoção de TIMs Fonte: Autores, com base no modelo original de Freitas e Machado (2009).

Em relação aos benefícios apresentados com a adoção de TIMs no projeto da empresa Alpha, estão listados: a diminuição do tempo em que os colaboradores ficarão afastados de suas atividades funcionais, a redução do cansaço e estresse causado pelo grande deslocamento e trânsito, aumento da motivação pelo uso de novos recursos e tecnologias. Estes benefícios, baseados no posicionamento também de Freitas e Machado (2009) vão ao encontro dos benefícios esperados com o uso de ferramentas colaborativas, as chamadas mídias sociais.

Sabe-se que as mídias sociais possuem potenciais benefícios para o ambiente organizacional, como ganho de produtividade, aumento de eficácia nas tarefas de rotina, redução de custos, remodelagem de processos, aproveitamento da inteligência coletiva para a 
tomada de decisão, criação de conteúdo de forma dinâmica, geração de conhecimento de forma colaborativa e visualização holística de todo o projeto (Filev, 2008; Bonabeau, 2009; Kosalge \& Tole, 2010; McDonald, 2011). Portanto, a escolha de mídias sociais para o referido processo, neste caso o Webex e a Rede Social Corporativa, poderão potencializar os benefícios esperados.

O presente relato limita-se pela impossibilidade de medir os resultados obtidos com o planejamento sugerido, pelo fato do projeto em questão ainda estar em execução pela empresa Alpha durante o momento da elaboração deste trabalho. Estudos futuros poderiam aplicar o modelo original, incluindo as melhorias aqui sugeridas, seja no ambiente de projetos ou qualquer, de forma a empiricamente validar ou contrapor os pontos observados.

\section{Agradecimento}

O presente trabalho foi realizado com o apoio do CNPq e do Fundo de Apoio à Pesquisa - FAP/UNINOVE.

\section{Referências}

Albertin, A. L. (2001). Valor estratégico dos projetos de tecnologia de informação. Revista de Administração de Empresas - RAE, São Paulo, v.41, n.3, p. 42-50.

Andriessen, J. H. E., \& Vartiainen, M. (Eds.). (2010). Mobile virtual work: a new paradigm? Berlin - Heidelber: Springer

Bautzer, D. (2009). Inovação: repensando as organizações. São Paulo: Atlas.

Biancolino, C., Kniess, C., Maccari, E., \& Rabechini, R., Jr. (2012). Protocolo para elaboração de relatos de produção técnica. GeP - Revista de gestão de projetos. v.3, n. 2, pp. 294-307.

Bonabeau, E. (2009). Decisions 2.0: the power of collective intelligence. MIT Sloan Management Review, v.50, n.2, p. 45.

Carvalho, M. M., \& Rabechini, R., Jr. (2010). Fundamentos em gestão de projetos. Construindo competências para gerenciar projetos. 3 ed. São Paulo: Atlas.

Ebrahim, N. A., Ahmed, S., \& Taha, Z. (2009). Virtual Teams: a Literature Review. Australian Journal of Basic and Applied Sciences, v.3, n.3, pp. 2653-2669.

Filev, A. (2008). Project management 2.0: the ultimate benefits of the new approach to project management. PM World Today, v.X. 
Freitas, H. M. R., \& Machado, C. B. (2009). Planejamento de iniciativas de adoção de tecnologias móveis. GEPROS - Gestão da produção, operações e sistemas. Ano 4, n.1, pp. 101-115.

ISO, A. N. (2012). 21500: 2012. Guidance on Project Management.

Kalakota, R., \& Robinson, M. (2002). M-business: tecnologia móvel e estratégia de negócios (p. 249). Porto Alegre: Bookman.

Katzenbach, J. R., \& Smith, D. K. (2005). The discipline of teams. Harvard Business Review, v.71, n.2, pp. 111-120.

Kosalge, P., \& Tole, O. (2010). Web 2.0 and business: early results on perceptions of web 2.0 and factors influencing its adoption. In AMCIS, p. 208.

McDonald, P. (2011). It's time for management 2.0: six forces redefining the future of modern management. Futures, v. 43, pp. 797-808.

Moore, G. C., \& Benbasat, I. (1991). Development of an instrument to measure the perceptions of adopting an information technology innovation. Information Systems Research, v.2, n.3, pp. 192-222.

Patton, M. Q. (2005). Qualitative research: Encyclopedia of statistics in behavioral science. v.3, pp. 1633-1636. Chichester: John Wiley \& Sons.

PMI Standards Committee. (2013). A guide to the project management body of knowledge - PMBoK. 5th Edition. Project Management Institute.

Rogers, E. M. (2003). Diffusion of innovations. $5^{\text {th }}$ Edition. New York: The Free Press.

Saccol, A. Z., \& Reinhard, N. (2007). Tecnologias de informação móveis, sem fio e ubíquas: definições, estado da arte e oportunidades de pesquisa. Revista de administração contemporânea. v.11, n.2, pp. 175-198.

Sandelowski, M. (2000). Focus on research methods: whatever happened to qualitative description? Research in nursing \& health. v.23, pp. 334-340.

Sorensen, C. (2011). Enterprise mobility: tiny technology with global impact on work. Hampshire: Palgrave Macmillan.

Thamhain, H. J. (2012). The changing role of team leadership in multinational project environments. GEP - Revista de Gestão de Projetos, v.3, n.2, pp. 4-38.

Yin, R. K. (2014). Case study research: design and methods. 5th ed., pp. 3-24. USA: Sage Publication. 
Van Aken, J. E. (2007). Developing organization studies as an applied science using a triple learning approach. Disponível em:

http://www.egosnet.org/organization_studies/summer_workshops/2007

Venkatesh, V., \& Davis, F. D. (2000). A theoretical extension of the technology acceptance model: four longitudinal field studies. Management Science, v.46, n. 2, pp. 186204.

Venkatesh, V., Morris, M. G., Davis, G. B., \& Davis, F. D. (2003). User aceptance of information technology: toward a unified view. Management Information Systems Quarterly - MISQ, v.27, n. 3, pp. 425-478. 